\title{
Fontes e níveis de metionina em dietas para frangos de corte ${ }^{1}$
}

\section{Maurício Tárcio dos Santos Viana ${ }^{2}$, Luiz Fernando Teixeira Albino ${ }^{3}$, Horacio Santiago Rostagno ${ }^{3}$, Sergio Luiz de Toledo Barreto ${ }^{3}$, Débora Cristine de Oliveira Carvalho², Paulo Cezar Gomes ${ }^{3}$}

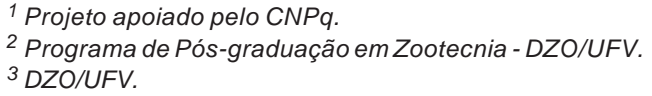

RESUMO - Objetivou-se avaliar o efeito de duas fontes de metionina, DL-metionina e metionina hidroxi análoga (MHA-AL), e de três níveis de suplementação desse aminoácido em dietas comerciais sobre o desempenho de frangos de corte. Foram utilizados 1.232 pintos de corte machos da linhagem Ross, de 1 a 47 dias de idade, distribuídos em delineamento inteiramente casualizado, em esquema fatorial $2 \times 3$ (fonte de metionina $\times$ nível de suplementação) e uma dieta adicional (basal) sem suplementação, com oito repetições de 22 aves por unidade experimental. A dieta basal (sem suplementação) foi suplementada com 50, 100 e 150\% de MHA ou DL-metionina em quantidade equivalente a 65\% da quantidade de cada nível de MHA-AL utilizado. Avaliaram-se o consumo de ração, o ganho de peso, a conversão alimentar e o fator de produção. Em cada nível de suplementação, não houve diferença entre as dietas contendo 100 partes de MHA-AL e 65 partes de DL-metionina, exceto no período de 1 a 10 dias de idade, quando houve diferença significativa no ganho de peso no menor nível de suplementação. Sessenta e cinco partes de DL-metionina equivalem a 100 partes de MHA-AL.

Palavras-chave: aminoácidos sulfurados, bioeficácia, desempenho, fontes de metionina, frangos de corte

\section{Methionine sources and levels in broiler chick diets}

\begin{abstract}
The objective of this study was to evaluate the effect of two sources of methionine, DL-Methionine (DLM - 99\%) and Methionine Hydroxy Analogue Free Acid (MHA-FA - 88\%), at three different levels of supplementation of this amino acids in commercial diets on the performance of broiler chickens. A total of 1.232 commercial Ross broilers was used, from 01 to 47 days of age, and allotted to a complete randomized design in a $2 \times 3$ factorial (source methionine $\times$ supplementation level) arrangement and a additional diet (basal) without supplementation, with eight replications of 22 birds per experimental unit. The basal diet was supplemented with 50, 100 and 150\% of MHA-FA or DL-methionine in equivalent amount of $65 \%$ of the amount of MHA-FA used. Feed intake, weight gain, feed:gain ratio and production factor were determined. Within each supplementation level there were no significant differences between the diets containing 100 parts of MHA-FA and 65 parts of DL-methionine, except for the period of 01-10 days when there was significant difference for the weight gain at the lowest level of supplementation. In conclusion, 65 parts of DL-methionine is equivalent to 100 parts of MHA-FA.
\end{abstract}

Key Words: biological efficacy, broiler chicks, methionine source, performance, sulphur amino acids

\section{Introdução}

As dietas práticas são formuladas principalmente à base de milho e farelo de soja e suprem as necessidades de proteína, mas não atendem às exigências de metionina e aminoácidos sulfurados, o que pode ser corrigido com a utilização de metionina sintética, que onera em aproximadamente 5\% o custo final da ração (Rodrigues et al., 1996).

A metionina, juntamente com a cisteína em dietas para aves, são os primeiros aminoácidos limitantes; logo a concentração adequada desses aminoácidos é fundamental para determinar que outros aminoácidos sejam utilizados com eficiência na síntese de proteína.

Na suplementação de metionina, normalmente são utilizadas duas fontes alternativas; DL-metionina, na forma de pó; e metionina hidroxi análoga em ácido livre (MHA-AL), na forma líquida (Weerden \& Schutte, 1984). A importância da efetividade biológica dessas fontes tem sido tema de debate entre nutricionistas, uma vez que o conhecimento desse parâmetro pode assegurar melhor desempenho 
zootécnico e econômico dos animais. Para tomar a decisão correta sobre qual fonte de metionina é mais efetiva, de acordo com o seu custo, os nutricionistas avaliam o potencial da MHA-AL em relação à DL-metionina.

Portanto, saber o valor nutritivo da MHA-AL em comparação à DL-metionina é importante para determinação do custo na compra de ingredientes, para formulação de dietas e para a produção animal. Em geral, a resposta do animal a um nutriente limitante como a metionina segue a lei dos retornos mínimos, ou seja, o desempenho animal melhora de forma não-linear com o aumento da suplementação dietética de metionina até que o potencial do animal, sob as condições de manejo a que está submetido, seja totalmente expresso e a maior adição de metionina não promova qualquer resposta adicional em desempenho.

Assim, com base nesta relação de resposta à dose, a especificação de metionina para rações comerciais de aves e de suínos deve ser estabelecida com cuidado visando obter o desempenho animal almejado durante o período. O nível adequado de metionina depende da finalidade de produção, da genética, da idade do animal, do ambiente e dos custos de produção. Portanto, é evidente a necessidade de se estabelecer o nível adequado de metionina e avaliar o efeito da substituição de DL-metionina por MHA-AL em dietas para frangos de corte considerando a biodisponibilidade de 65\% da MHA-AL.

\section{Material e Métodos}

O experimento foi conduzido no Setor de Avicultura do Departamento de Zootecnia da Universidade Federal de Viçosa. Foram utilizados 1.232 pintos de corte machos da linhagem Ross no período de 1 a 47 dias de idade, alojados em galpão de alvenaria coberto com telhas de cerâmica francesa, provido de lanternim, com piso de cimento e subdividido em 56 boxes de 1,0 × 1,5 m com cama de maravalha. O fornecimento de ração e de água foi à vontade durante todo o período experimental. As temperaturas foram registradas diariamente, por meio de termômetros de máxima e mínima localizados em um ponto estratégico à altura das aves.

As aves foram distribuídas em esquema fatorial $2 \times 3$ (fonte de metionina $\times$ nível de suplementação), com oito repetições por dieta e 22 aves por unidade experimental. Uma dieta basal (sem suplementação de metionina) foi suplementada com 50, 100 e 150\% de MHA-AL (Tabela 1) ou DL-metionina em quantidade equivalente a $65 \%$ da quantidade de cada nível de MHA-AL utilizado (Tabela 2).

As dietas foram formuladas com milho, farelo de soja e subprodutos de origem animal, segundo recomendações da empresa integradora. Foram formuladas dietas basais para as fases pré-inicial, inicial e de crescimento/final. A composição da dieta final foi igual à da dieta de crescimento (Tabela 3), retirando-se apenas os aditivos (anticoccidiano e promotor de crescimento).

Avaliaram-se o consumo de ração, o ganho de peso, a conversão alimentar e o fator de produção. O consumo médio de ração foi calculado em quilogramas (kg), pela diferença entre a ração fornecida e a sobra do comedouro, registrando-se o consumo da ração por unidade experimental no final de cada período. O ganho de peso, expresso em gramas, foi calculado como a diferença entre os pesos inicial e final das aves em cada unidade experimental. A conversão alimentar foi obtida dividindo-se o consumo médio da ração pelo ganho de peso médio das aves de cada unidade experimental. $\mathrm{O}$ fator de produção, ou índice de eficiência produtiva, foi determinado aos 47 dias, de acordo com a fórmula: [(ganho de peso $(\mathrm{kg}) \times$ viabilidade (\%) / idade (dias) $\times$ conversão alimentar) $] \times 100$.

As análises estatísticas foram feitas por meio de análise de variância (ANOVA), com subsequente teste de média Student Newman Keuls (SNK), utilizando-se o programa

Tabela 1 - Representação das estratégias de suplementação

\begin{tabular}{|c|c|c|c|c|c|c|}
\hline \multirow[b]{2}{*}{$\begin{array}{l}\text { Fonte de } \\
\text { metionina }\end{array}$} & \multirow[b]{2}{*}{$\begin{array}{c}\text { Nível de } \\
\text { suplementação }\end{array}$} & \multicolumn{2}{|c|}{ Suplementação relativa } & \multicolumn{3}{|c|}{ Suplementação de metionina (kg/t de ração) } \\
\hline & & $\begin{array}{c}\text { Nível } \\
\text { comercial }\end{array}$ & $\begin{array}{l}\text { Fonte de } \\
\text { metionina }\end{array}$ & 1 a 10 dias & 11 a 28 dias & 29 a 47 dias \\
\hline Sem suplementação & Basal & $0 \%$ & - & - & - & - \\
\hline MHA-AL & Nível 1 & $50 \%$ & $100 \%$ & 1,84 & 1,50 & 1,04 \\
\hline DL-metionina & & - & $65 \%$ & 1,20 & 0,98 & 0,68 \\
\hline MHA-AL & Nível 2 & $100 \%^{1}$ & $100 \%$ & 3,68 & 2,99 & 2,06 \\
\hline DL-metionina & & - & $65 \%$ & 2,39 & 1,94 & 1,34 \\
\hline MHA-AL & Nível 3 & $150 \%$ & $100 \%$ & 5,52 & 4,49 & 3,10 \\
\hline DL-metionina & & - & $65 \%$ & 3,59 & 2,92 & 2,02 \\
\hline
\end{tabular}

${ }^{1}$ Nível de suplementação utilizado nas dietas comerciais. 
SAEG (UFV, 1999). Foram feitas duas ANOVAS: na primeira, o objetivo foi realizar contrastes entre a média da dieta basal e as médias das dietas com MHA-AL e DL-metionina; e, na segunda análise estatística, foram usados somente as dietas do fatorial $2 \times 3$ (fonte de metionina $\times$ nível de suplementação).

Tabela 2 - Dietas experimentais e níveis de suplementação de MHA e DL-metionina

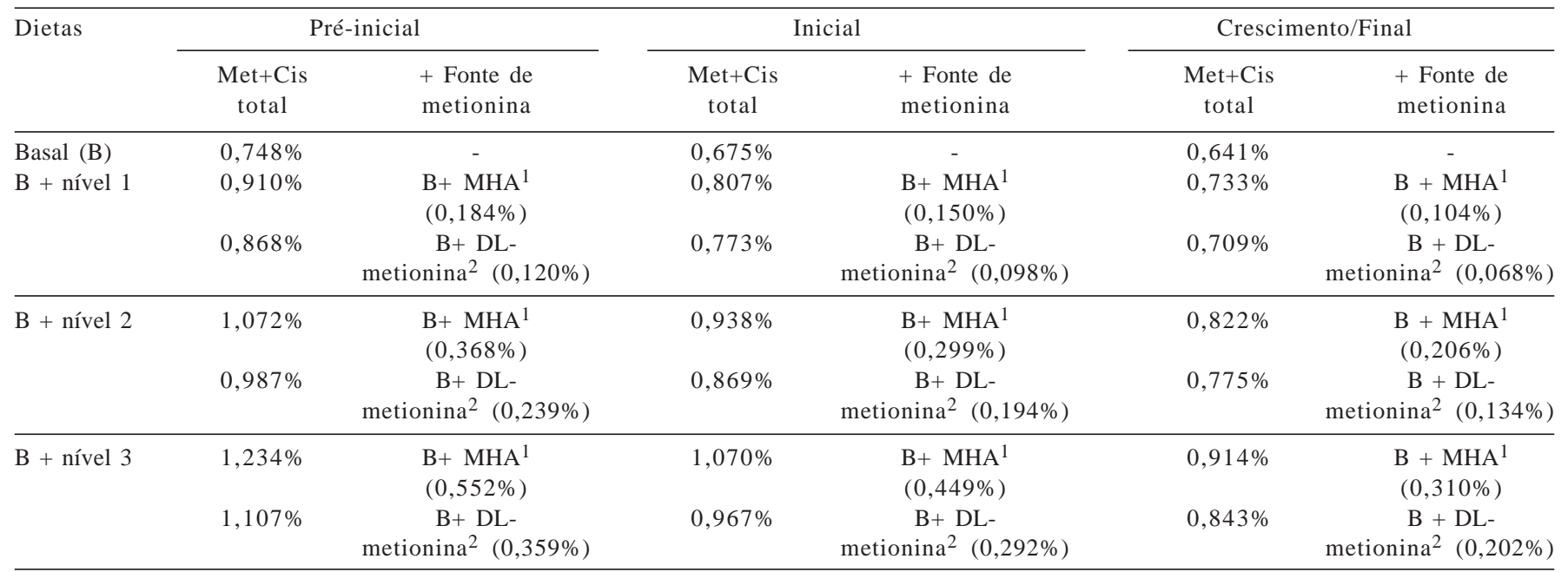

${ }^{1}$ MHA considerada com bioequivalência de $88 \%$.

2 DL-metionina adicionada na relação de $65 \%$ da MHA.

Tabela 3 - Composição das dietas basais

\begin{tabular}{|c|c|c|c|}
\hline Ingrediente (\%) & $\begin{array}{l}\text { Pré-inicial } \\
\text { (1 a } 10 \text { dias) }\end{array}$ & $\begin{array}{c}\text { Inicial } \\
\text { (11 a } 28 \text { dias })\end{array}$ & $\begin{array}{l}\text { Crescimento } \\
\text { (29 a } 42 \text { dias) }\end{array}$ \\
\hline Milho & 48,528 & 55,151 & 68,735 \\
\hline Farelo de soja & 29,900 & 22,200 & 14,700 \\
\hline Soja integral tostada & 15,525 & 15,825 & 4,850 \\
\hline Farinha de carne & 3,675 & 3,875 & 2,300 \\
\hline Farinha de penas & - & - & 2,000 \\
\hline Farinha de vísceras & - & - & 4,000 \\
\hline Gordura de aves & - & 1,000 & 1,500 \\
\hline Sal comum & 0,500 & 0,500 & 0,400 \\
\hline Calcário (38\%) & 0,900 & 0,612 & 0,675 \\
\hline Mistura mineral $^{1}$ & 0,050 & 0,050 & 0,050 \\
\hline Mistura vitamínica² & 0,100 & 0,100 & 0,100 \\
\hline BioLys 65 (50,7\% de lisina) & 0,084 & 0,059 & 0,220 \\
\hline Cloreto de colina & 0,066 & 0,060 & 0,055 \\
\hline Anticoccidiano & 0,055 & 0,055 & 0,055 \\
\hline Natuphos (fitase) & 0,010 & 0,005 & 0,005 \\
\hline Avilamicina $(10 \%)$ & 0,007 & 0,007 & 0,007 \\
\hline Amido $^{3}$ & 0,600 & 0,500 & 0,350 \\
\hline \multicolumn{4}{|l|}{ Composição calculada } \\
\hline Energia metabolizável, kcal/kg & 3.010 & 3.150 & 3.250 \\
\hline Proteína bruta ${ }^{4}, \%$ & 24,89 & 22,00 & 19,00 \\
\hline Cálcio, \% & 1,00 & 0,90 & 0,90 \\
\hline Fósforo disponível, \% & 0,50 & 0,50 & 0,45 \\
\hline Lisina total ${ }^{4}, \%$ & 1,45 & 1,24 & 1,02 \\
\hline Lisina digestível, \% & 1,27 & 1,08 & 0,88 \\
\hline Met+Cis total ${ }^{4}, \%$ & 0,748 & 0,675 & 0,641 \\
\hline
\end{tabular}

${ }^{1}$ Níveis de suplementação mineral: manganês - 65 g; ferro - 50,0 g; zinco - 60,0 g; cobre - 10,0 g; selênio - 0,30 g; iodo - 0,8 g.

2 Níveis de suplementação vitamínica: vit. A - 10.000 UI; vit. $\mathrm{D}_{3}$ - 2.000 UI; vit. E - 35 UI; vit. $\mathrm{B}_{1}-1,5 \mathrm{mg}$; vit. $\mathrm{B}_{6}$ - 2,4 mg; àcido pantotênico - 12,0 mg; biotina - 0,070 mg. vit. $\mathrm{K}_{3}$ - 1,7 mg; ácido fólico - 0,700 mg; ácido nicotínico - 35,0 g; vit. $\mathrm{B}_{12}$ - 0,012 mg; selênio - 0, $33 \mathrm{mg}$.

${ }^{3}$ Usado para corrigir as adições de MHA e DL-metionina. A MHA e DL-metionina substituíram o amido das rações basais para originar as dietas suplementadas com metionina.

${ }^{4}$ Valores calculados e confirmados pelas análises das dietas experimentais. 


\section{Resultados e Discussão}

Os valores médios diários das temperaturas máxima e mínima ao longo do período experimental foram, respectivamente, de 22,3 e $27,4^{\circ} \mathrm{C}$. Nenhuma diferença ( $P>0,01)$ foi encontrada no consumo de ração entre os níveis, as fontes e a interação fonte $\times$ nível de metionina. As aves mantidas com a dieta sem suplementação de metionina (dieta basal) apresentaram menor consumo de ração em todas as fases, portanto, foi significativo $(\mathrm{P}<0,01)$ em comparação às demais dietas (Tabelas 4, 5 e 6). Esses resultados corroboram os de Brittes (1990), que também não encontraram efeito de níveis de suplementação de DL-metionina sobre o consumo de ração.

Em cada nível de suplementação, não houve diferenças $(\mathrm{P}>0,01)$ entre as dietas contendo MHA-AL e DL-metionina, exceto no período de 1 a 10 dias de idade, no qual houve diferença significativa para o ganho de peso no menor nível de suplementação (Tabela 5). Comparando duas fontes de metionina (DL-metionina e MHA-AL) e diversos níveis de suplementação desse aminoácido sobre o desempenho de frangos de corte de 1 aos 47 dias de idade, Meirelles et al.(2003) não encontraram diferença entre as fontes ou os níveis nem interação entre eles. Entretanto, observaram em todas as fases aumento do ganho de peso de acordo com o nível de metionina.

Dentro de cada nível de suplementação, as dietas não promoveram diferenças $(\mathrm{P}>0,01)$ na conversão alimentar, evidenciando que as duas fontes de metionina estudadas são igualmente eficientes para o crescimento do animal. A adição de MHA-AL e DL-metionina melhorou $(\mathrm{P}<0,01)$ a conversão alimentar em relação à dieta basal em todas as fases estudadas. Resultados similares foram encontrados por Buresh \& Harms (1986), que trabalharam com duas fontes de metionina e não encontraram diferença entre elas.

No entanto, Meirelles et al (2003) encontraram melhor conversão alimentar em aves alimentadas com dietas suplementadas com DL-metionina. A conversão alimentar também melhorou com o aumento dos níveis de suplementação de metionina nas dietas.

Não se observou diferença $(\mathrm{P}>0,01)$ no consumo de ração no período total. Entretanto, as aves que receberam dietas suplementadas com DL-metionina apresentaram maiores valores absolutos para o consumo de ração nos níveis intermediário e superior. Resultados semelhantes foram encontrados por Buresh \& Harms (1986), Schutte \& Weerden (1987) e Bastiani et al. (1994), que não encontraram diferenças estatísticas no consumo de ração ao compararem duas fontes de metionina (DL-metionina e MHA-AL).

Rostagno \& Barboza (1995), trabalhando com frangos de corte na fase de 21 a 42 dias de idade em ambiente de estresse por calor, demonstraram que o consumo de ração não foi influenciado pelas dietas experimentais. Neste estudo, no entanto, o consumo de ração entre as aves que receberam dietas suplementadas com DL-metionina foi menor que o daquelas alimentadas com as rações suplementadas com MHA-AL. Todavia, Schutte \& Pack(1995) observaram redução da ingestão alimentar com o aumento dos níveis de metionina e dos aminoácidos sulfurados na dieta de frangos de corte.

As aves alimentadas com dietas de menor nível de suplementação (nível 1) apresentaram desempenho estatisticamente $(\mathrm{P}<0,01)$ inferior aos daquelas que receberam rações com os níveis intermediários e superiores de metionina. Aumento crescente do ganho de peso com o acréscimo dos níveis de metionina na dieta também foi observado por Schutte \& Weerden (1981) e Schutte et al. (1983). Entretanto, Schutte \& Weerden (1987), Thomas et al. (1991) e Weerden et al. (1992) encontraram resultados significativamente inferiores para a MHA-AL em relação à DL-metionina.

Houve resposta para a conversão alimentar aos níveis de suplementação das fontes de metionina e o efeito benéfico

Tabela 4 - Desempenho de frangos de corte de 1 a 10 dias de idade alimentados com rações suplementadas com metionina

\begin{tabular}{|c|c|c|c|c|c|c|c|c|c|}
\hline \multirow[b]{2}{*}{ Fonte e Nível } & \multicolumn{3}{|c|}{ Consumo ração (kg) } & \multicolumn{3}{|c|}{ Ganho de peso (kg) } & \multicolumn{3}{|c|}{ Conversão alimentar } \\
\hline & MHA & DL-metionina & Média & MHA & DL-metionina & Média & MHA & DL-metionina & Média \\
\hline Basal (B) & 0,211 & $* *$ & & 0,1 & ** & & & $9 * *$ & \\
\hline B + Nível 1 & 0,297 & 0,269 & 0,283 & $0,211 \mathrm{a}$ & $0,195 b$ & $0,203 B$ & 1,413 & 1,379 & $1,396 \mathrm{~B}$ \\
\hline B + Nível 2 & 0,298 & 0,296 & 0,297 & $0,226 a$ & $0,229 a$ & $0,228 \mathrm{~A}$ & 1,317 & 1,293 & $1,305 \mathrm{~A}$ \\
\hline B + Nível 3 & 0,296 & 0,296 & 0,296 & $0,224 \mathrm{a}$ & $0,221 \mathrm{a}$ & $0,223 \mathrm{~A}$ & 1,322 & 1,335 & $1,329 \mathrm{~A}$ \\
\hline Média & 0,297 & 0,287 & & 0,220 & 0,215 & & 1,351 & 1,336 & \\
\hline
\end{tabular}

$\begin{array}{llll}\text { CV (\%) } \quad 9,84 & 3,74 & 4,81\end{array}$

** Contraste da dieta basal versus média MHA e média DL-metionina significativo $(\mathrm{P}<0,01)$.

a, b Médias nas linhas seguidas por diferentes letras diferem $(\mathrm{P}<0,01)$ pelo SNK.

A, B Médias nas colunas seguidas por diferentes letras diferem $(\mathrm{P}<0,01)$ pelo SNK. 
Tabela 5 - Desempenho de frangos de corte de 1 a 28 dias de idade alimentados com rações suplementadas com metionina

\begin{tabular}{|c|c|c|c|c|c|c|c|c|c|}
\hline \multirow[b]{2}{*}{ Fonte e Nível } & \multicolumn{3}{|c|}{ Consumo ração (kg) } & \multicolumn{3}{|c|}{ Ganho de peso (kg) } & \multicolumn{3}{|c|}{ Conversão alimentar } \\
\hline & МHA & DL-metionina & Média & MHA & DL-metionina & Média & МHA & DL-metionina & Média \\
\hline Basal (B) & 1,540 & $* *$ & & 0,84 & $6 * *$ & & 1,8 & $2 * *$ & \\
\hline B + Nível 2 & 2,024 & 2,018 & 2,021 & 1,328 & 1,315 & $1,322 \mathrm{~A}$ & 1,524 & 1,534 & $1,529 \mathrm{~A}$ \\
\hline B + Nível 3 & 2,028 & 1,987 & 2,008 & 1,332 & 1,334 & $1,333 \mathrm{~A}$ & 1,524 & 1,486 & $1,505 \mathrm{~A}$ \\
\hline
\end{tabular}

** Contraste da dieta basal versus média MHA e média DL-metionina significativo $(\mathrm{P}<0,01)$.

A, B Médias nas colunas seguidas por diferentes letras diferem $(\mathrm{P}<0,01)$ pelo SNK.

Tabela 6 - Desempenho de frangos de corte de 1 a 47 dias de idade alimentados com rações suplementadas com metionina

\begin{tabular}{|c|c|c|c|c|c|c|c|c|c|}
\hline \multirow[b]{2}{*}{ Fonte e Nível } & \multicolumn{3}{|c|}{ Consumo ração $(\mathrm{kg})$} & \multicolumn{3}{|c|}{ Ganho de peso $(\mathrm{kg})$} & \multicolumn{3}{|c|}{ Conversão alimentar } \\
\hline & МHA & DL-metionina & Média & МНА & DL-metionina & Média & MHA & DL-metionina & Média \\
\hline Basal (B) & 4,292 & ** & & & $4 * *$ & & & $3 * *$ & \\
\hline B + Nível 1 & 5,438 & 5,246 & 5,342 & 2,716 & 2,676 & $2,696 \mathrm{~B}$ & 2,002 & 1,962 & $1,982 \mathrm{~B}$ \\
\hline B + Nível 2 & 5,335 & 5,381 & 5,358 & 2,823 & 2,842 & $2,833 \mathrm{~A}$ & 1,890 & 1,894 & $1,892 \mathrm{~A}$ \\
\hline B + Nível 3 & 5,367 & 5,426 & 5,397 & 2,852 & 2,895 & $2,874 \mathrm{~A}$ & 1,882 & 1,876 & $1,879 \mathrm{~A}$ \\
\hline Média & 5,380 & 5,351 & & 2,797 & 2,804 & & 1,925 & 1,911 & \\
\hline
\end{tabular}

$\begin{array}{llll}\mathrm{CV}(\%) & 3,86 & 3,80 & 2,79\end{array}$

** Contraste da dieta basal versus média MHA e média DL-metionina significativo $(\mathrm{P}<0,01)$.

A, B Médias nas colunas seguidas por diferentes letras diferem $(\mathrm{P}<0,01)$ pelo SNK.

foi mais evidente nos níveis de 100 e $150 \%$ de suplementação de MHA-AL. No entanto, o desempenho das aves não diferiu $(\mathrm{P}>0,01)$ entre os níveis 2 e 3 . Rostagno \& Barboza (1995) observaram que a adição de MHA-AL e DL-metionina melhorou significativamente a conversão alimentar em relação à dieta basal, mas entre elas não houve diferença significativa. Resultado semelhante foi apresentado por Schuttte \& Pack (1995).

Dentro de cada nível de suplementação (Tabela 7), não houve diferenças $(\mathrm{P}>0,01)$ entre as dietas quanto ao fator de

Tabela 7 - Valores médios do fator de produção de 1 a 47 dias de idade

\begin{tabular}{llcc}
\hline Fonte e Nível & MHA & DL-metionina & Média \\
\hline Basal (B) & \multicolumn{4}{c}{$190,76 * *$} & & \\
B + Nível 1 & 262,97 & 264,60 & $263,79 B$ \\
B + Nível 2 & 289,50 & 290,80 & 290,15A \\
B + Nível 3 & 290,90 & 299,18 & 295,04A \\
\hline Média & 281,12 & 284,86 & \\
\hline CV (\%) & 5,36 & \\
\hline
\end{tabular}

** Contraste da dieta basal vs média MHA e média DL-metionina significativo $(\mathrm{P}<0,01)$.

A, B Médias nas colunas seguidas por diferentes letras diferem $(\mathrm{P}<0,01)$ pelo SNK. produção. Houve, no entanto, resposta do fator de produção aos níveis de suplementação das fontes de metionina e efeito benéfico mais evidente nos níveis de 100 e $150 \%$ de MHA-AL, que não diferiram $(\mathrm{P}>0,01)$ entre si.

\section{Conclusões}

Nos níveis avaliados, as fontes de metionina DLmetionina e MHA-AL são equivalentes na alimentação de frangos de corte.

\section{Literatura Citada}

BASTIANI M.F.; COSTA, P.T.C.; RUBIN, M.A. et al. Avaliação de três fontes de metionina a dois níveis de adição no desempenho de frangos de corte. In: CONFERÊNCIA APINCO DE CIÊNCIA E TECNOLOGIA AVÍCOLAS, 1994, Santos. Anais... Santos: Fundação Apinco de Ciência e Tecnologia Avícolas, 1994. p.177-178.

BRITTES, L.B.P. Efeito de níveis de DL-Metionina sobre o desempenho e composição química do tecido muscular em frangos de corte no período de 1-49 dias de idade. 1990. 93f. Dissertação (Mestrado em Zootecnia) - Universidade Federal de Santa Maria, Santa Maria, 1990.

BURESH, R.E.; HARMS, R.H. Comparison of DL-Methionine and Alimet ${ }^{\circledR}$ whit broilers when full fedor restricted. Nutrition Reports International, v.33, n.3, p.449-457, 1986. 
MEIRELLES, H.T.; ALBUQUERQUE, R.; BORGATTI, L.M.O. et al. Performance of broilers fed with different levels of methionine hydroxy analogue and DL-methionine. Brazilian Journal of Poultry Science, v.5, p.69-74, 2003.

RODRIGUES, P.B.; BETERCHINI, A.G.; OLIVEIRA, B.L. et al. Fatores nutricionais que influenciam a qualidade do ovo no segundo ciclo de produção. Níveis de aminoácidos sulfurosos totais. Revista Brasileira de Zootecnia, v.25, n.2, p.248-260, 1996.

ROSTAGNO, H.S.; BARBOZA, W.A. Biological efficacy and absorption of DL-methionine hydroxy analogue free acid compared to DL-methionine in chickens as affected by heat stress. British Poultry Science, v.36, p.303-312, 1995.

SCHUTTE, J.B.; WEERDEN, E.J. Effectiveness of DL-methionine hydroxy analogue in comparison with DL-methionine in broilers. Feedstuffs, v.53, n.26, p.16, 1981.

SCHUTTE, J.B.; WEERDEN, E.J.; BERTRAN, H.L. Sulphur amino acid requirement of laying hens and the effects of excess dietary methionine on laying performance. British Poultry Science, v.24, p.319-326, 1983
SCHUTTE, J.B.; PACK, M. Sulfur amino acid requirement of broiler chick from fourteen to thirty eight days of age. Performance and carcass yield. Poultry Science, v.74, p.480-487, 1995.

SCHUTTE, J.B.; WEERDEN E.J. Effectiveness of methionine hydroxy analogue as affected by the dietary level of L-methionine in chicks. Nutrition Reports International, v.36, n.2, p.253-259, 1987.

THOMAS, O.P.; TAMPLIN, C.; CRISSEY, S.D. et al. An evaluation of methionine hydroxy analogue free acid using a non linear exponential bioassay. Poultry Science, v.70, p.605-610, 1991.

UNIVERSIDADE FEDERAL DE VIÇOSA - UFV. Sistema de análises estatísticas e genéticas - SAEG. Versão 8.0. Viçosa, MG, 1999. 59p. (Manual do usuário).

WEERDEN, E.J.; SCHUTTE, J.B., BERTRAN, H.L. Utilization of the polymers of methionine analogue free acid (MHA-FA) in broilers chicks. Arch Gefliigclkd, v.56, p.63-68, 1992.

WEERDEN, E.J.; SCHUTTE, J.B. Comparison of DL-methionine, DL-methionine-Na, DL-methionine hydroxy analogue-Ca, and DL-methionine hydroxy analogue free acid with layers. Poultry Science, v.63, p.1793-1799, 1984. 